\title{
Stochastic stability and the evolution of coordination in spatially structured populations
}

\author{
Jeremy Van Cleve ${ }^{\mathrm{a}, *}$, Laurent Lehmann ${ }^{\mathrm{b}}$ \\ a Santa Fe Institute, 1399 Hyde Park Road, Santa Fe, NM 87501, USA \\ ${ }^{\mathrm{b}}$ Department of Ecology and Evolution, University of Lausanne, Le Biophore, CH-1015, Lausanne, Switzerland
}

\section{H I G H L I G H T S}

- We apply stochastic stability to the evolution of coordination.

- Increased population structure leads to higher payoff outcomes even when risky.

- Large social groups lead to anti-coordination when competition is strong.

\section{A R T I C L E I N F O}

Article history:

Received 14 May 2013

Available online 30 August 2013

\section{Keywords:}

Risk dominance

Relatedness

n-player game

Stag hunt

\begin{abstract}
A B S T R A C T
Animals can often coordinate their actions to achieve mutually beneficial outcomes. However, this can result in a social dilemma when uncertainty about the behavior of partners creates multiple fitness peaks. Strategies that minimize risk ("risk dominant") instead of maximizing reward ("payoff dominant") are favored in economic models when individuals learn behaviors that increase their payoffs. Specifically, such strategies are shown to be "stochastically stable" (a refinement of evolutionary stability).

Here, we extend the notion of stochastic stability to biological models of continuous phenotypes at a mutation-selection-drift balance. This allows us to make a unique prediction for long-term evolution in games with multiple equilibria. We show how genetic relatedness due to limited dispersal and scaled to account for local competition can crucially affect the stochastically-stable outcome of coordination games. We find that positive relatedness (weak local competition) increases the chance the payoff dominant strategy is stochastically stable, even when it is not risk dominant. Conversely, negative relatedness (strong local competition) increases the chance that strategies evolve that are neither payoff nor risk dominant. Extending our results to large multiplayer coordination games we find that negative relatedness can create competition so extreme that the game effectively changes to a hawk-dove game and a stochastically stable polymorphism between the alternative strategies evolves. These results demonstrate the usefulness of stochastic stability in characterizing long-term evolution of continuous phenotypes: the outcomes of multiplayer games can be reduced to the generic equilibria of two-player games and the effect of spatial structure can be analyzed readily.
\end{abstract}

(c) 2013 Elsevier Inc. All rights reserved.

\section{Introduction}

Social behavior occurs across all domains of life, from colonies of microscopic prokaryotes to swarms of insects and herds of ungulates, and generates some of the most complex biological phenomena (e.g., ant supercolonies and human societies). Given this complexity, understanding the evolutionary forces that shape social behavior has been a persistent focus of evolutionary theory.

\footnotetext{
* Correspondence to: National Evolutionary Synthesis Center (NESCent), $2024 \mathrm{~W}$. Main Street Suite A200, Durham, NC 27705, USA.

E-mail addresses: vancleve@nescent.org (J. Van Cleve), laurent.lehmann@unil.ch (L. Lehmann).
}

Much of this focus has been devoted to exploring which conditions are favorable for the evolution of helping behaviors such as cooperation or altruism in public goods or prisoner's dilemma games and their repeated game counterparts.

Although these scenarios are manifestly important, they do not characterize all situations in which cooperation is useful but potentially disfavored by natural selection. One such situation occurs when animals must coordinate their actions to achieve a mutually beneficial outcome, such as when predators can cooperate to hunt prey. For example, some populations of wild chimpanzees hunt colobus monkeys in groups (Boesch, 1994, 2002), and individuals in the group obtain more payoff than those who do not hunt (Boesch, 1994). This suggests that cooperating in the hunt may be an evolutionary stable strategy (ESS). However, if 
none of the chimpanzees are hunting, then it does not benefit any single individual to hunt alone, which suggests that not hunting is also a candidate ESS.

Social situations can thus have multiple possible candidate ESSs and the underlying games are often called "coordination games" (Binmore, 2007) or "stag hunt games" (Skyrms, 2001) given their similarity to a scenario described by the philosopher Jean-Jacques Rousseau (Crawford, 1991). In the classic description of the stag hunt, hunters must cooperate to hunt stag, a valuable prey item, but can also hunt hare, easier but less valuable prey, on their own. The inherent dilemma in the stag hunt and other coordination games is that individuals may not coordinate on hunting stag or whatever strategy provides the highest payoff for all individuals at the ESS (also known as a "payoff dominant" strategy). Thus, the evolutionary problem is to determine what biological circumstances, if any, yields an evolutionary dynamic that lead to the payoff dominant outcome.

Because there are two candidate ESSs in the stag hunt game, the evolutionary dynamics of hunting hare or stag will depend on the history or initial conditions of the process, and in a very large population local fitness maxima (ESS points) will tend to deterministically attract the evolutionary dynamic. However, random effects introduced by mutations and genetic drift occurring in small populations may allow the population to escape from one local equilibrium and to shift towards the other peak. Previous work shows that this exploration process resulting from the interaction of mutation, selection, and drift will often find local fitness peaks that are "risk dominant" (Harsanyi and Selten, 1988), even if such peaks are not payoff dominant and do not provide the highest payoff to all individuals (Blume, 1993; Ellison, 1993; Kandori et al., 1993; Young, 1993). A focal strategy is risk dominant over an alternative strategy when an individual obtains more payoff from the focal strategy on average given it has no information about which of the two strategies its partner will choose. When the effect of mutation and drift become very small, this stochastic exploration process results in the population residing at the less "risky" peak nearly all the time, and this peak is consequently called "stochastically stable" (Foster and Young, 1990). In the case of a coordination game or stag hunt, this would suggest that individuals would always hunt hare instead of stag if hunting hare were less risky regardless of how much meat a stag might yield.

Despite the importance of demographic and genetic population structure in the evolution of public goods, its effect on the evolution of social dilemmas in coordination games under continuously varying strategies is almost entirely unexplored. The primary goal of the present paper is to incorporate genetic structure and relatedness into an explicit stochastic model for the evolution of such strategies. In order to do this, we will extend the concept of stochastic stability used in evolutionary game theory to continuous phenotypes in structured populations. We find that positive relatedness leads to a greater opportunity for the payoff dominant strategy to be selected instead of the risk dominant one; that is, more relatedness tends to favor more reward rather than less risk. We extend this model to $n$-player interactions and derive novel results on how the evolution of coordination works as interaction groups increase in size. Specifically, we find that negative relatedness, which translates to intense competition between kin, can change the nature of the game itself when interaction groups are large. Instead of the evolution of risk dominant strategies, negative relatedness in such groups results in the evolution of a strategic polymorphism, which is the result of effective hawk-dove type interactions. Thus, we find that natural selection does not simply result in the evolution of less risky strategies; instead, it can select alternative high payoff strategies when relatedness is positive and can create stochastically stable polymorphisms when relatedness is strongly negative.

\section{Model}

\subsection{Biological assumptions}

\subsubsection{Population setting}

We consider a group structured population of constant and finite size $N_{\mathrm{T}}=N n_{\mathrm{d}}$, where $n_{\mathrm{d}}$ is the number of groups and $N$ the number of individuals in a group. The spatial structure of the population may follow a variety of schemes, such as the island model of dispersal (Wright, 1931), the standard isolation-by-distance model of population genetics where individuals (or groups) live on a lattice (Malécot, 1975), or a hierarchically clustered model such that individuals are grouped into families, families into villages, villages into clans, and so on (Sawyer and Felsenstein, 1983).

The individuals in the population may be iteroparous or semelparous, but we leave the exact details of the life history and population structure (i.e., the dispersal kernel) unspecified as it does not affect our analysis. All that matters is that interactions between individuals can occur at a local scale, among group members or among nearest neighbors on a lattice, instead of occurring at random in the whole population.

\subsubsection{Social interactions}

We assume that the individuals in the population participate in a social game where they must select one of two actions, $r$ or $p$. The probability that individual $i$ chooses, or "plays", action $r$ is denoted by $z_{i}$. We assume that the payoff (taken here to be average fecundity relative to a baseline) to individual $i$ when meeting individual $j$ that plays action $r$ with probability $z_{j}$ is given by

$$
\begin{aligned}
f\left(z_{i}, z_{j}\right)= & 1+z_{i}\left[z_{j} R+\left(1-z_{j}\right) S\right] \\
& +\left(1-z_{i}\right)\left[z_{j} T+\left(1-z_{j}\right) P\right],
\end{aligned}
$$

where $R$ can be thought of as the reward for mutual cooperation, $S$ the sucker's payoff, $T$ the temptation to defect, and $P$ the punishment for mutual defection. When $R>T$ and $P>S$, the payoff structure represents a coordination or stag hunt game (Skyrms, 2001), so that interacting individuals have an incentive to both play either $r$ or $p$.

\subsubsection{Evolutionary dynamics}

Our aim is to derive the long-term evolutionary dynamic of the play probability $z$ in the population. To do this, we assume that the phenotype $z$ is determined by a single genetic locus with a continuum of possible allelic effects (Kimura, 1965; Bürger, 2000). We also make the standard assumption of evolutionary game theory, adaptive dynamics, and inclusive fitness theory that the stochastic dynamics of $z$ allow for only two segregating alleles at a time, a resident allele and a mutant allele (e.g., Eshel, 1996; Hammerstein, 1996; Metz et al., 1996; Eshel et al., 1998; Geritz et al., 1998; Rousset, 2004; Champagnat et al., 2006; Champagnat and Lambert, 2007). This assumption is justifiable if the per capita mutation rate $\mu$ to new alleles is small relative to the total population size (specifically when $\mu N_{\mathrm{T}} \log \left(N_{\mathrm{T}}\right) \ll 1$; Champagnat et al., 2006; Champagnat and Lambert, 2007). It has its origin in population genetics where it has been widely applied in molecular evolution to evaluate substitution rates (e.g., Kimura, 1971; Gillespie, 1991) and used to evaluate the steadystate fitness landscape in the absence of social interactions (Iwasa, 1988; Gillespie, 1991; Sella and Hirsh, 2005; Sella, 2009).

In order to evaluate how selection, drift, and mutation interact in the presence of social interactions in a population with only mutant and resident alleles, we look at individuals carrying a mutant allele to play action $r$ with probability $z+\delta$ in a population where resident (wild-type) individuals play action $r$ with probability $z$. 
Hence, $\delta$ is the phenotypic deviation in the play probability by mutant individuals. The values of $\delta$ are drawn from a symmetric distribution with mean zero, which means that mutations do not tend to push the phenotype in one direction or another. We assume that $\delta$ is small (weak selection) so that the evolutionary dynamics can be evaluated to first order in $\delta$. With all these assumptions and regardless of the kind of population structure, the probability density function $p(z, t)$ of observing a population monomorphic for phenotype $z$ at time $t$ on an long-term evolutionary time scale satisfies the diffusion equation

$\frac{\partial p(z, t)}{\partial t}=-\mu \sigma^{2} N_{\mathrm{T}} \frac{\partial}{\partial z}[\delta(z) p(z, t)]+\frac{\mu \sigma^{2}}{2} \frac{\partial^{2}}{\partial z^{2}}[p(z, t)]$,

where $\sigma^{2}$ is the variance in the mutation step size (variance in $\delta$ ), and $s(z)$ is the derivative of the fixation probability of a single mutant with respect to $\delta$ evaluated in a population where the resident trait $z$ is at fixation (i.e., $\delta=0$; see Lehmann, 2012, Eq. (4)). The term $\delta(z)$ determines the mean change in the phenotype at that point and determines the direction of selection in a population fixed for $z$. Fluctuations around the mean path are described by the second term in Eq. (2).

The term $s(z)$ can be thought of as a "selection gradient" and can be expressed as an inclusive fitness effect as it provides a demographically explicit version of Hamilton's (1964) inclusive fitness effect for finite populations (Rousset, 2004; Rousset and Ronce, 2004; Rousset, 2006). The inclusive fitness effect is a relatedness weighted sum of the effects of individuals expressing the mutant behavior on the expected number of offspring of individuals carrying the mutant allele. Relatedness captures the frequency of mutant-mutant interactions, which result from local correlations in allele frequency in different groups in the population. In the absence of such interactions, a panmictic population for example, $s(z)$ reduces to the selection gradient used in the adaptive dynamics literature for finite populations (Champagnat and Lambert, 2007, p. 5; for a proof see Lehmann, 2012, Eq. (A-24)). In the limit of large population size $\left(N_{\mathrm{T}} \rightarrow \infty\right), s(z)$ becomes proportional to standard phenotypic selection gradients used in evolutionary game theory, adaptive dynamics, and inclusive fitness theory (Rousset, 2004).

\subsection{Stationary, convergence, and stochastically stable states}

In the infinite time limit $(t \rightarrow \infty)$, the forces of selection, mutation, and drift acting on the evolving phenotype eventually balance each other out to yield a stationary probability density of observing phenotype value $z, p(z)=\lim _{t \rightarrow \infty} p(z, t)$. The standard solution of the diffusion equation in $(2)$ in this limit and with reflecting boundaries can be written as (Karlin and Taylor, 1981; Ewens, 2004; Gardiner, 2009)

$p(z)=K \exp \left[2 N_{\mathrm{T}} \phi(z)\right]$,

where $K$ denotes a normalizing constant,

$\phi(z)=\int \delta(z) \mathrm{d} z$.

The function $\phi(z)$ is a so-called potential function since its derivative with respect to $z, s(z)$, determines the direction of change of the phenotype (Gardiner, 2009).

The stationary distribution may have several peaks (maxima) and valleys (minima). We call the set of all peaks $\mathcal{C}$. These peaks correspond to phenotypes that are more likely to occur in the long run than their neighboring phenotypes in trait space. Because the stationary distribution is monotonically increasing as a function of $\phi(z)$, the set of the highest peaks of the stationary distribution, $\mathcal{M} \in \mathcal{C}$, can be obtained directly by maximizing the potential:
$\mathcal{M}=\left\{y: \phi(y)=\max _{z \in Z} \phi(z)\right\}$. The set $\mathcal{M}$ includes several peaks only if they are of exactly equal height. These highest, or global, peaks are more likely to be observed in the long run of the evolutionary dynamics than any of the local peaks.

A useful feature of the stationary density $p(z)$ is that its peaks, $\mathcal{C}$, characterize the convergence stable states (Lehmann, 2012), which are equivalent to the classic definition of convergence stability in evolutionary theory (Eshel, 1983; Lessard, 1990; Christiansen, 1991; Rousset, 2004; Leimar, 2009). A convergence stable phenotype is one where mutants are selected to invade a population of residents when their phenotype is closer to the convergence stable phenotype than the resident phenotype. In terms of the selection gradient $s(z)$, a convergence stable phenotype $z$ (not on the boundaries of the trait space) satisfies the conditions $\delta(z)=0$ and $\mathrm{d} s(z) / \mathrm{d} z<0$ (Rousset, 2004), which are equivalent to the conditions required for $z$ to be a local maximum of $p(z)$, namely $\mathrm{d} p(z) / \mathrm{d} z=0$ and $\mathrm{d}^{2} p(z) / \mathrm{d} z^{2}<0$. A convergence stable phenotype may also lie on the boundary of the trait space in which case (if the above condition does not apply) the condition is $8(z)<0$ for the lower boundary or $\delta(z)>0$ for the upper boundary, which corresponds to a boundary maximum for $p(z), \mathrm{d} p(z) / \mathrm{d} z<0$ or $\mathrm{d} p(z) / \mathrm{d} z>0$. Thus, the more common phenotypes in the stationary distribution, $\mathcal{C}$, correspond exactly to the convergence stable phenotypes obtained from an application of the inclusive fitness effect or the invasion fitness used in evolutionary theory.

The existence of multiple convergence stable states suggests that different populations might evolve to different states if these populations have different initial phenotypes that reside in different basins of attraction. Over long evolutionary time scales, stochastic forces may shift populations out of the basin of attraction of one convergence stable state to another, and this peak shifting process equilibrates to the stationary density $p(z)$ such that the population spends more time at some convergence stable states than others. A biological and mathematically useful feature of this peak shifting process is that as stochastic forces become negligible (population size becomes large), a small set of convergence stable states (often just one state) remain visited with some frequency and all other states are unvisited. Specifically, we will say that $z$ is stochastically stable if

$\lim _{N_{\mathrm{T}} \rightarrow \infty} p(z)>0$.

This definition of stochastic stability follows from previous work in economics and game theory that studies the long run behavior of populations of game playing agents that update their strategies from discrete sets using simple learning rules (Foster and Young, 1990; Kandori et al., 1993; Noldeke and Samuelson, 1993; Binmore et al., 1995; Sandholm, 2010). In these models, a stochastically stable state is one retaining positive probability in a vanishing noise limit, which in practice is obtained by either letting population size become large or mutation rate go to zero (Foster and Young, 1990; Kandori et al., 1993; Noldeke and Samuelson, 1993; Binmore et al., 1995; Sandholm, 2010).

In Appendix A, we prove that for our continuously varying state space, all stochastically stable states satisfy

$z \in \mathcal{M}$.

Hence, a stochastically stable state is a global maximum of $p(z)$. Importantly, the stochastic stable state can be determined from knowing the selection gradient $s(z)$ alone (Eqs. (4) and (6)).

The result (6) parallels for continuously varying strategies a standard result for stochastic stability on discrete state spaces (Foster and Young, 1990; Kandori et al., 1993; Binmore et al., 1995; Sandholm, 2010). The main message behind the notion of stochastic stability is that it reduces the set of candidate evolutionary 
states to a single point, which is the global maximum of the stationary distribution. Thus, it gives a unique prediction for the longterm behavior of the evolutionary dynamics in a population of very large size. We will now employ this notion in order to analyze the evolution of the play probabilities in a coordination game with and without spatial population structure.

\subsubsection{Fixation probability perturbation}

From the preceding section, we know that the crucial quantity we need to calculate in order to understand the long-term stochastic evolutionary dynamics of the play probability $z$ is the derivative of the fixation probability with respect to the mutant deviation $\delta$, namely $\delta(z)$. In order to evaluate this quantity explicitly, we use standard results for the evolution of continuous phenotypes in structured populations of finite size (Rousset, 2004). Let $z$. denote the phenotype of a focal individual in the population (which could be either mutant or resident) and denote by $z_{0}$ the average phenotype of individuals (excluding the focal) in the group of the focal individual. We also assume that only the focal individual itself and its group members (or nearest neighbors on a lattice) can affect the payoff (fertility or survival) of the focal individual.

With these assumptions, we can write the derivative of the fixation probability as

$$
s(z)=\left.\frac{k(z)}{f(z, z)}\left(\frac{\partial f\left(z_{\bullet}, z_{0}\right)}{\partial z_{\bullet}}+\kappa(z) \frac{\partial f\left(z_{\bullet}, z_{0}\right)}{\partial z_{0}}\right)\right|_{z_{\bullet}=z_{0}=z} .
$$

This expression consists of two terms, which can be interpreted as follows. First, there is the effect of the focal individual on its own number of offspring produced, which stems from it changing its own play probability. Second, there is a "relatedness" weighted effect that a randomly sampled group member (different than the focal) has on the number of offspring produced by the focal individual when the group member changes its play probability.

The coefficient $\kappa$ is a scaled relatedness coefficient that includes both local competition effects (e.g., Queller, 1994) due to limited local resources and global effects due to the finiteness of the population, dispersal limitation, and other effects of population structure. The coefficient $\kappa$ depends on life cycle parameters (e.g., population structure, life-history modes) and can be positive, negative, or zero within the interval $[-1,1]$ (Lehmann and Rousset, 2010). Likewise, the constant of proportionality $k$ also depends on life cycle properties. Under non-Poisson distributed fecundity or when the evolving trait directly affects demographic parameters like dispersal, $k$ and $\kappa$ will both depend on $z$. This can lead to dynamical interactions between effects of $z$ on payoff and on population structure. While Eq. (7) is expressed in terms of fecundity effects of social behavior, it can also be modified to include survival effects. In the general case, $k$ and $\kappa$ will both almost invariably depend on survival as it critically affects the genealogical structure of populations.

The form of the selection gradient (Eq. (7)) follows generally and mechanically from the assumption of weak selection (neglecting terms $O\left(\delta^{2}\right)$, which here also implies additive gene action). This linearizes the fitness function that determines fixation probabilities (Rousset, 2003, 2004), so that the vital rate $v$ of a focal individual (fecundity or survival) depends on a linear combination of the derivatives of $v$ with respect to the phenotype of the focal and the phenotypes of other homogeneous classes of social partners. Each derivative is weighted by a coefficient that captures the effect of demographic structure on that type of individual, which may themselves depend on vital rates (we refer to Rousset, 2004 for details on the general construction of game theoretic models in spatially structured populations). When there is only one class of social partner affecting the vital rate of a focal recipient, the fixation probability can be factored as in Eq. (7) and written in term of the average phenotype of that class, otherwise one would need to account for a scaled relatedness coefficient for each class of social partners (e.g., Lehmann et al., 2007a, Eq. (8)).

The message behind Eq. ( 7$)$ is that in order to obtain $s(z)$ for multiplayer games, one essentially needs only to specify the fertility (or survival) of an individual as a function of its own behavior and the average of its neighbors. This decomposition of the selection gradient into perturbations on vital rates occurs explicitly or implicitly in a large number of previous models of social evolution in both finite and infinite populations. For instance, in the recent literature $\kappa$ is equivalent to the "potential for altruism" of Gardner (2010, p. 340), the "potential for helping" of Rodrigues and Gardner (2012, Eq. (4)), the "compensated relatedness" of Grafen and Archetti (2008, p. 696), the scaled relatedness of Van Dyken (2010, Eq. (4)) and Akcay and Van Cleve (2012, p. 258), the " $\sigma_{0}$ " of Alger and Weibull (2012), or the " $\sigma$ " of Tarnita et al. (2009) and Allen et al. (2013). More generally, $\kappa$ has been evaluated under a large class of life history and demographic scenarios. In Appendix B, we present various examples of $\kappa$ and $k$ values for the classical island and isolation-by-distance models of population structure of finite size for both fecundity and survival effects; because $\kappa$ critically affects the selection pressure on $z$, we summarize the analytical results for $\kappa$ in Table 1. For a more comprehensive presentation of how an expression like Eq. (7) relates to different demographic scenarios in populations of very large total size, we refer to Lehmann and Rousset (2010).

Given the diversity of possible life history conditions in natural populations, we assume that $\kappa$ is a fixed parameter and analyze the evolution of the play probability $z$. We thus assume that both $k$ and $\kappa$ do not depend on the evolving trait and remain nonzero when population size becomes large $\left(N_{\mathrm{T}} \rightarrow \infty\right)$; in practice, this implies that we focus on social traits with fecundity effects where fecundity is either Poisson distributed or infinitely large, as is assumed in many evolutionary analyses.

\section{Analysis}

\subsection{Pairwise coordination game}

\subsubsection{Stationary distribution}

Substituting the payoff function (1) into the fixation probability perturbation (Eq. (7)), we have

$s(z)=\frac{k}{f(z, z)}(S-P+z D+\kappa[T-P+z D])$

where $D=R-S-T+P$. There are three potential local stable states: (i) $z=1$ where individuals choose action $r$ with probability 1; (ii) $z=0$ where individuals choose action $r$ with probability 0 ; and (iii) a mixed strategy where individuals choose action $r$ with the intermediate value

$z^{*}=\frac{P-S+\kappa(P-T)}{(1+\kappa) D}$,

which is obtained by solving $s\left(z^{*}\right)=0$. The mixed strategy $z^{*}$ is plotted in the left panel of Fig. 1 . When $\kappa=0$, the mixed strategy is the standard one from two-player games in infinite populations with deterministic dynamics, and when $\kappa=-1 /\left(N_{\mathrm{T}}-1\right)$ it reduces to the mixed strategy for finite panmictic populations (Schaffer, 1988; Wild and Taylor, 2004).

Substituting Eq. (8) into Eq. (4) produces the potential function

$\phi(z)=\frac{k}{2}[(1+\kappa) \log (X(z))-(1-\kappa) Y(z)]$

where

$X(z)=\frac{1+P+(R-P) z-D z(1-z)}{1+P}$ 
Table 1

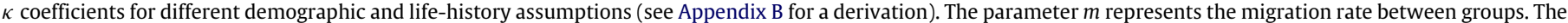

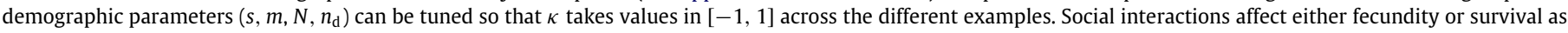
indicated.

\begin{tabular}{|c|c|}
\hline Scaled-relatedness coefficient $\kappa$ & Case \\
\hline$-\frac{1}{N_{\mathrm{T}}-1}$ & $\begin{array}{l}\text { Interaction among all members in a panmictic population, whether semelparous or iteroparous and whether social effects are } \\
\text { on fecundity or survival. }\end{array}$ \\
\hline$-\frac{1}{N_{\mathrm{T}}-1}$ & $\begin{array}{l}\text { Interactions within groups in an arbitrarily structured homogeneous population with semelparous reproduction and large } \\
\text { offspring number (Wright-Fisher process with only fecundity effects). }\end{array}$ \\
\hline$-\frac{1}{N-1}$ & $\begin{array}{l}\text { Interactions within groups in an arbitrarily structured homogeneous population with semelparous reproduction and population } \\
\text { size regulation before dispersal. }\end{array}$ \\
\hline$\frac{(1-m)-2 / n_{\mathrm{d}}}{[N+(1-m)]-2 / n_{\mathrm{d}}}$ & $\begin{array}{l}\text { Interactions within groups in an island model of dispersal with iteroparous reproduction and one death per generation (Moran } \\
\text { process with fecundity effects). }\end{array}$ \\
\hline$-\frac{1}{N_{\mathrm{T}}-1}$ & $\begin{array}{l}\text { Interactions within groups in an island model of dispersal with iteroparous reproduction and one death per generation (Moran } \\
\text { process with survival effects). }\end{array}$ \\
\hline$\frac{q-\left[2 q / n_{\mathrm{d}}+m /\left(N n_{\mathrm{d}}\right)\right]}{m\left(N n_{\mathrm{d}}-1\right) /\left(N n_{\mathrm{d}}\right)+q\left(N+n_{\mathrm{d}}-2\right) / n_{\mathrm{d}}}$ & Interactions within groups and group fission at rate $q$ with competition between groups to form the next generation. \\
\hline$\frac{m-2 k / n_{\mathrm{d}}}{k(2-m)-2 k / n_{\mathrm{d}}}$ & $\begin{array}{l}\text { Interactions among } k \text {-nearest neighbors on a lattice under stepping-stone dispersal and iteroparous reproduction (Moran } \\
\text { process with fecundity effects). }\end{array}$ \\
\hline$-\frac{1}{N_{\mathrm{T}}-1}$ & $\begin{array}{l}\text { Interactions among } k \text {-nearest neighbors on a lattice under stepping-stone dispersal and iteroparous reproduction (Moran } \\
\text { process with survival effects). }\end{array}$ \\
\hline$\frac{s[2-m(1+s)] / 2-(1+s) / n_{\mathrm{d}}}{N+s[2-m(1+s)] / 2-(1+s) / n_{\mathrm{d}}}$ & $\begin{array}{l}\text { Interactions within groups in an arbitrarily structured homogeneous population with weak migration ( } m \ll 1 \text { ), iteroparous } \\
\text { reproduction, and survival probability } s \text { of an individual to the next generation (fecundity effects). }\end{array}$ \\
\hline$-\frac{(1-s)[2-m(1+s)] / 2+(1+s) / n_{\mathrm{d}}}{2 N-(1-s)[2-m(1+s)] / 2-(1+s) / n_{\mathrm{d}}}$ & $\begin{array}{l}\text { Interactions within groups in an arbitrarily structured homogeneous population with weak migration }(m \ll 1 \text { ), iteroparous } \\
\text { reproduction, and survival probability } s \text { of an individual to the next generation (survival effects). }\end{array}$ \\
\hline
\end{tabular}
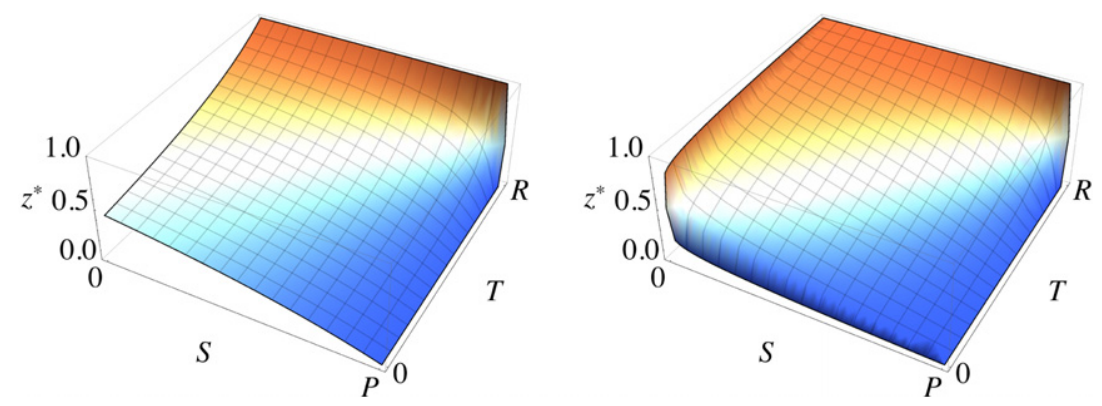

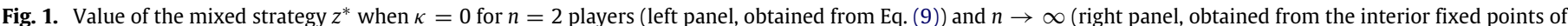

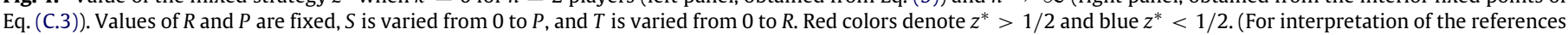
to colour in this figure legend, the reader is referred to the web version of this article.)

$$
\begin{aligned}
Y(z)= & \frac{2(T-S)}{V}\left[\tan ^{-1}\left(\frac{R-P-D(1-2 z)}{V}\right)\right. \\
& \left.+\tan ^{-1}\left(\frac{P-R+D}{V}\right)\right]
\end{aligned}
$$

with $V=\sqrt{4(1+P)(1+R)-(2+S+T)^{2}}$. Using this expression for $\phi(z)$ in Eq. (3) allows us to study the long-term probability that the population is fixed for play probability $z$.

\subsubsection{Stochastic stability}

In the case of a coordination game $(R>S$ and $R>T$, where, without loss of generality, we further set $R>P$ ), there are two convergence stable states $(z=1$ and $z=0$, whereby $\mathcal{C}=$ $\{1,0\})$, and we are interested in whether populations tend to have phenotypes that choose action $r$ more often than $p$, or vice versa.

We borrow the terminology of Fudenberg et al. (2006) and say that action $r$ is "favored" when it is chosen more often than $p$, which implies that $\mathrm{E}[z]>1 / 2$ under the stationary distribution, where $\mathrm{E}[z]=\int_{0}^{1} z p(z)$ is the mean phenotype. In the limit as population size becomes very large $\left(N_{\mathrm{T}} \rightarrow \infty\right)$, the favored strategy is "selected" in the sense that the probability density becomes a delta function located at one of the convergence stable states, either $z=1$ when $r$ is selected or $z=0$ when $p$ is selected. The selected strategy is the stochastically stable state of the system. In the case of a coordination game, $z=1$ is stochastically stable when $\phi(1)>\phi(0)$ and $z=0$ is stochastically stable when $\phi(1)<\phi(0)$.
From Eq. (10), we have $\phi(0)=0$, and $z=1$ is thus a stochastically stable state if $\phi(1)>0$. Exponentiating both sides of this inequality, using Eq. (10), and noting that $X(1)=(1+R) /(1+$ $P)$, playing only action $r(z=1)$ is the stochastically stable state if

$$
\left(\frac{1+\kappa}{1-\kappa}\right) \log \left(\frac{1+R}{1+P}\right)>Y(1) .
$$

Hence, holding everything else constant, the larger the value of $\kappa$, the more likely it is that the action $r$ is stochastically stable. Since the payoff when both individuals choose $r$ is higher than when they both choose $p$ (i.e., $R>P$ ), larger values of $\kappa$ make it more likely that the payoff dominant action is stochastically stable.

\subsubsection{Payoff vs. risk dominance}

While individuals are better off in terms of the number of offspring produced when everybody expresses the payoff dominant action $r$, this does not mean that evolution proceeds towards that action. It has been repeatedly shown that when individuals learn to play actions in stochastic models, the dynamics do not proceed towards the payoff dominant action (Young, 1993; Kandori et al., 1993; Fudenberg and Harris, 1992; Ellison, 1993); this implies the existence of a social dilemma in coordination games. Rather, the condition for an action to be stochastically stable in these models is that it is risk dominant.

If players are unsure of the strategy of their partner and assign a probability $1 / 2$ to each of their partner's actions, the expected payoff of playing the risk dominant action exceeds that of playing 

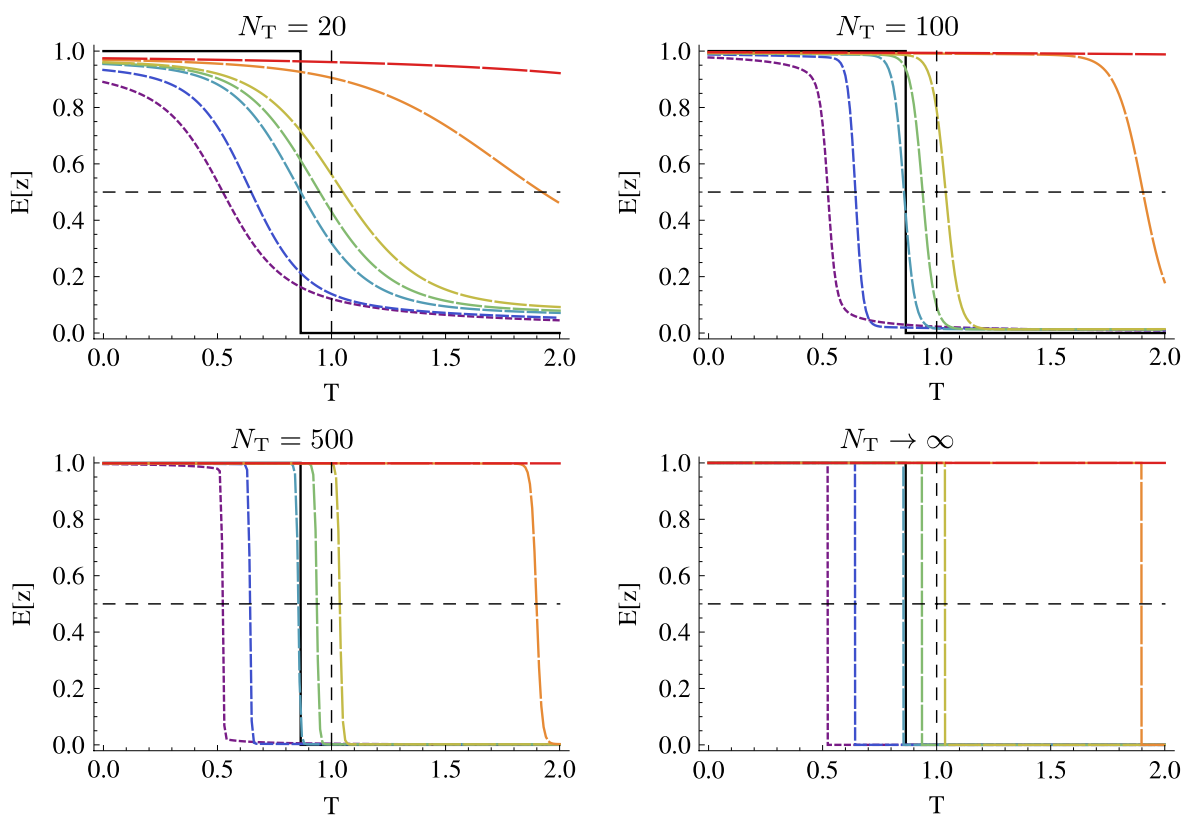

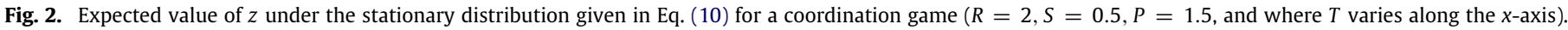

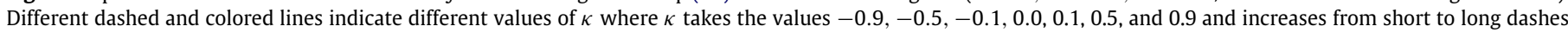

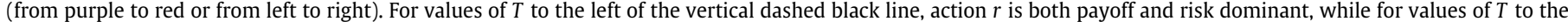

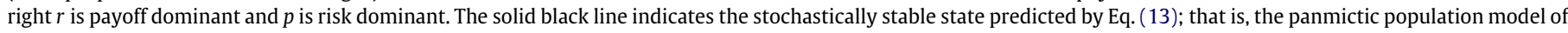

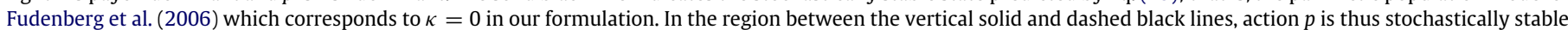

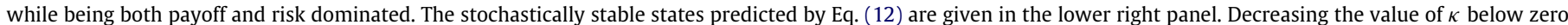

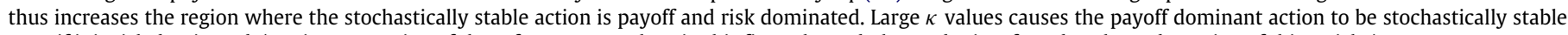
even if it is risk dominated. (For interpretation of the references to colour in this figure legend, the reader is referred to the web version of this article.)

the payoff dominant action (Kandori et al., 1993; Binmore et al., 1995). In the context of a single population whose evolutionary dynamic is described by the replicator equation (Taylor and Jonker, 1978; Hofbauer and Sigmund, 2003), the risk dominant action is the one with the larger basin of attraction. The size of the basins of attraction is determined by the value of the mixed strategy $z^{*}$ (obtained by solving $s\left(z^{*}\right)=0$ ), which is unstable (i.e., $f^{\prime}\left(z^{*}\right)>0$ ) under the replicator dynamics for the payoffs of a coordination game. The action with the larger basin of attraction is then the one farther from $z^{*}$. In terms of the payoffs, this means that action $p$ is risk dominant over action $r$ if $T-S>(R-P)(1+\kappa) /(1-\kappa)$ (i.e. if $z^{*}>1 / 2$ from Eq. (9)). The higher the value of $T$, the more likely action $r$ is to be risk dominated. In terms of hunting stag (action $r$ ) or hunting hare (action $p$ ), the value of $T$ can be interpreted as tuning the intensity of interference of individuals hunting stag on those hunting hare, which may occur when stag hunters are likely to destroy burrows of hares or frighten them away. When $T$ is increased, there is less interference of hunting stag on hare hunting and the latter becomes less risky. This can be seen visually in the left panel of Fig. 1, where $r$ is risk dominant for $z^{*}<1 / 2$ and $p$ is risk dominant for $z^{*}>1 / 2$.

Unlike the learning models in game theory, models grounded in population genetics have not always found that the risk dominant action is stochastically stable. For example, using a population genetic approach based on the Moran model for panmictic populations (Moran, 1958), Fudenberg et al. (2006) showed that neither the risk nor the payoff dominant action is stochastically stable for some parameter values. In Fudenberg et al., action $r$ is stochastically stable if

$\frac{R \log (R)-S \log (S)}{R-S}>\frac{T \log (T)-P \log (P)}{T-P}$

(Theorem 2 part b.3 of Fudenberg et al., 2006). Fig. 2 shows this condition (black line) for the case where the strategy $r$ is payoff dominant: $R=2, S=0.5, P=1.5$, and $T$ varies along the $x$-axis.
Values of $T$ greater than the vertical dashed black line indicate the $p$ strategy is risk dominant, whereas $r$ is risk dominant for $T$ to the left of the line. The figure shows that action $p$ is stochastic stable for values of $T$ between the solid and dashed black vertical lines despite being neither risk nor payoff dominant.

By evaluating the mean play probability, $\mathrm{E}[z]$, from the stationary distribution (Eq. (10)), we can evaluate whether or not risk dominance is the condition for stochastic stability in our continuous phenotype model and how that changes as a function of the scaled relatedness coefficient $\kappa$. In Fig. 2 in the fourth line from the left $(\kappa=0)$, we graph the mean phenotype $E[z]$ obtained from the stationary distribution (Eq. (10)) with the potential function for the coordination game (Eq. (3)). As population size increases, it is clear that our model reproduces the qualitative result of $\mathrm{Fu}-$ denberg et al. (2006). However, the region of $T$ in which action $p$ is stochastically stable despite being neither payoff nor risk dominant is smaller than predicted by Eq. (13). Although our approach takes only first-order effects of selection into account, Fudenberg et al. (2006) do not make this simplifying assumption. This suggests that the quantitative difference is likely to be explained by the fact that Fudenberg et al. (2006) consider pure strategies, while our model consider the evolution of mixed strategies, which entails that third-order genetic associations do not affect the dynamics. Nevertheless, the qualitative concordance indicates that even just first-order effects of selection are able to capture this important effect of finite population size and drift on coordination games.

The reason for this generic difference between risk dominance based on the deterministic replicator equation and stochastic stability is that the stochastic dynamics take into account not only the size of the basin of attraction of a strategy but also the "expected speed of the flow" (Fudenberg et al., 2006) at each point of the state space, which is determined by the magnitude of $s(z)$ (Eq. (2)). To see how the magnitude of $s(z)$ indicates that a stochastically stable phenotype may not be risk dominant, we plot in Fig. $3 s(z)$ along with its definite integral $\phi(z)$ (Eq. (4)) for parameters that 


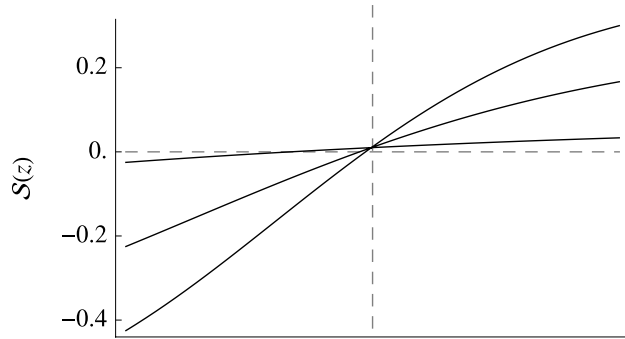

$(S, T)=(0.15,1.1)$

$(\mathrm{S}, T)=(0.55,1.5)$

$(S, T)=(0.95,1.9)$

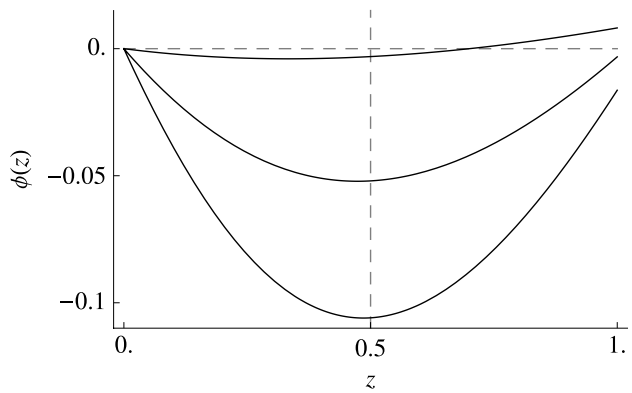

$(S, T)=(0.95,1.9)$

$(\mathrm{S}, T)=(0.55,1.5)$

$(S, T)=(0.15,1.1)$

Fig. 3. Selection gradient $s(z)$ given by Eq. (8) (top panel) and its definite integral $\phi(z)$ (bottom panel) for a semelparous panmictic population with $N_{\mathrm{T}} \rightarrow \infty$ ( $k$ and $\kappa$ given by Eq. (B.1)) where $R=2, P=1$, and the $S$ and $T$ values are given in the plots; these parameter values imply that $R+S>T+P$, which means $r$ is risk dominant (and payoff dominant). Although for the $(S, T)=(0.15,1.1)$ and $(S, T)=(0.55,1.5)$ lines, playing $r$ is risk dominant, we see that the selection gradient $s(z)$ takes more negative values (on the left of the graph) than positive values (on the right), which entails that $\phi(1)<0$ so that $r$ is stochastically unstable. The opposite trend is observed for the $(S, T)=(0.95,1.9)$ line in which case $\phi(1)>0$ and $r$ is stochastically stable.

make action $r$ risk dominant. Even though action $r$ is both risk and payoff dominant in the plots (i.e., has a larger basin of attraction), $|\delta(0)|>|\delta(1)|$ for $(S, T)=(0.15,1.1)$ and $(S, T)=(0.55,1.5)$, which suggests for these parameters that selection against action $r$ when it is played infrequently is stronger than selection for $r$ when played frequently. This results in very strong selection against action $r$ when rare and causes $\phi(1)<0$ for these values of $(S, T)$, and consequently action $p$ to be stochastically stable. For $(S, T)=$ $(0.95,1.9)$, selection for $r$ when played frequently increases enough relative to selection against $r$ when played infrequently that $\phi(1)$ becomes positive and $r$ becomes stochastically stable.

Fig. 2 also shows that increasing $\kappa$ increases the range of values of $T$ under which action $r$ is stochastically stable. This can be understood by noting that when a focal individual increases its play probability for action $r$, its neighbors are also likely to do so, as already alluded to above. This correlated effect, which stems from individuals having genes with similar effects on play probabilities due to relatedness, increases the selection pressure for $z=1$. From Eq. (8), one can infer that increasing $\kappa$ both increases the region of the state space where $z$ is selected for (on average) and the probability that $z$ increases when playing $r$ is selected against. Increasing $\kappa$ thus tilts the balance in favor of the payoff dominant action, and this occurs regardless of how it otherwise affects the game (see Eq. (12)). Hence, for large values of $\kappa$, the payoff dominant strategy is very likely to be selected for even if it is strongly risk dominated. For negative values of $\kappa$, the opposite trend is observed (Fig. 2).

\section{2. n-player coordination game}

\subsubsection{Game structure}

We have considered so far only pairwise interactions. However, social interactions often occur between more than two individuals. Thus, we may ask how the previous results concerning two-player coordination games change as the number of players increases.
Though general $n$-player games are difficult to analyze because they can exhibit complex dynamics and equilibrium structures (e.g., Motro, 1991; Bach et al., 2006; Gokhale and Traulsen, 2010; Peña, 2012), $n$-player interactions are considerably simpler using the population genetic assumptions described above (i.e., weak selection and weak mutation relative to $N_{\mathrm{T}}$ ). Specifically, to the first order in $\delta$, we can still consider $z_{\bullet}$ to be the probability that a focal individual plays action $r$, while $z_{0}$ is the play probability of action $r$ by a randomly sampled individual socially interacting with the focal individual. Hence, the payoff of the focal individual can still be written as a function of only $z_{\bullet}$ and $z_{0}$ (and Eq. (7) still holds), and the only difference with the previous situation is that the focal now interacts with $n-1$ other individuals, where each may play $r$ with probability $z_{0}$.

We assume that when the focal individual chooses action $r$, it obtains payoff $R$ if all other $n-1$ individuals also choose $r$. For every one of the $n-1$ other individuals who instead choose action $p$, the payoff of the focal individual, $R$, is decreased by a factor $\gamma<1$. In terms of hunting stag, this means that the likelihood of catching stag decreases as the participation in hunting decreases, and when $\gamma=0$, the stag can only be caught if all $n$ group members participate in the hunt. We also assume that when the focal individual chooses action $p$, it obtains payoff $P$ if all other $n-1$ individuals choose $p$, and for every one of the $n-1$ other individuals that chooses action $r$ when the focal individual chooses action $p$, the focal individual's payoff $P$ is scaled by a factor $\lambda$. In terms of hunting hare, this means that the likelihood of finding hares is a decreasing function of individuals hunting stag when $\lambda<1$ and is increasing as a function of the number hunting stag when $\lambda>1$ (less competition for finding hares from those who hunt stag). When $\lambda=0$, hares can be found only if all individuals participate in hare hunting. These assumptions lead to the expected payoff

$$
\begin{aligned}
f\left(z_{\bullet}, z_{0}\right)= & 1+R z_{\bullet}\left(z_{0}+\gamma\left(1-z_{0}\right)\right)^{n-1} \\
& +P\left(1-z_{\bullet}\right)\left(\lambda z_{0}+1-z_{0}\right)^{n-1}
\end{aligned}
$$

(see Eqs. (C.1)-(C.2) in Appendix C). In order to make the twoplayer and $n$-player games easily comparable, we choose $\gamma=$ $(S / R)^{1 /(n-1)}$ and $\lambda=(T / P)^{1 /(n-1)}$, which ensures that the payoffs to the focal are the same regardless of the number of players when the play probabilities $z_{\bullet}$ and $z_{0}$ are either zero or one. This means that, regardless of $n$, the focal obtains payoff $1+R$ when it and its partners always plays action $r, 1+S$ when it always plays $r$ and its partners always play $p$, and so on. This also ensures that Eq. (14) simplifies to Eq. (1) for the case of two players $(n=2)$ regardless of the play probabilities.

Substituting the payoff function (Eq. (14)) into the selection gradient (Eq. (7)) allows us to study the evolution of the play probability in the $n$-player coordination game in the presence or absence of population structure. Obtaining the stationary distribution $p(z)$ using the selection gradient is quite difficult analytically as it appears to involve the roots of $n$-th order polynomials. We thus proceed numerically to obtain the stationary distribution of $z$ and integrate the stationary distribution to obtain the mean phenotype $\mathrm{E}[z]$. Before presenting these results, we generalize the definitions of payoff and risk dominance to $n$-player interactions.

\subsubsection{Payoff vs. risk dominance}

In $n$-player interactions, payoff dominance naturally generalizes from the two-player case in that strategy $r$ is payoff dominant over strategy $p$ if $R>P$. In order to characterize risk dominance, we determine the mixed strategy $z^{*}$ for $\kappa=0$ (see Appendix C):

$z^{*}=\frac{P^{\frac{1}{n-1}}-S^{\frac{1}{n-1}}}{R^{\frac{1}{n-1}}-S^{\frac{1}{n-1}}-T^{\frac{1}{n-1}}+P^{\frac{1}{n-1}}}$, 
which simplifies to the correct mixed strategy for the two-player case. Given this value of $z^{*}$, action $r$ has a larger basin of attraction and is risk dominant when

$R^{\frac{1}{n-1}}+S^{\frac{1}{n-1}}>T^{\frac{1}{n-1}}+P^{\frac{1}{n-1}}$.

As the number of players grows very large and $n \rightarrow \infty, z^{*}$ becomes

$z^{*}=\frac{\log (P)-\log (S)}{\log (R)-\log (S)-\log (T)+\log (P)}$

and the condition for $r$ to be risk dominant becomes $R S>T P$. This is equivalent to saying that $r$ is risk dominant when the geometric mean payoff of $r$ is higher than that of $p$ when the focal individual assumes its $n-1$ partners will either all choose $r$ or $p$ with equal probability. The difference in the $n \rightarrow \infty$ and $n=2$ risk dominance conditions is due essentially to the assumption above that the deviations of the actions of the social partners from the action of the focal affect the payoff of the focal multiplicatively (see Appendix C).

Fig. 4 plots the mean play probability $\mathrm{E}[z]$ for $N_{\mathrm{T}}=500$ for $n=2,5,50$, and $n \rightarrow \infty$ using the same payoffs as in Fig. 2 . For more than two players and $\kappa=0$, we see that the region in which action $p$ is selected for even though it is neither payoff nor risk dominant disappears. Rather, a small region appears for $T$ larger than the threshold given in (16) for which $p$ is risk dominant but $r$ is still selected. This suggests that the benefit of payoff dominant strategies may increase as the size of the interaction group increases.

As in the two-player case, Fig. 4 shows that increasing $\kappa>0$ increases the range of $T$ for which the action $r$ is stochastically stable; this is true even as the number of players grows very large. A different pattern emerges however for strongly negative values of $\kappa$. Once there are more than two players, Fig. 4 suggests that a threshold value of $\kappa<0$ exists for which sufficiently small $T$ results in neither action, $r$ or $p$, being selected in the long run. Rather, an intermediate value of the play probability is expected, $0<\mathrm{E}[z]<1$. In order for an intermediate value of $z$ to be selected, neither $z=0$ nor $z=1$ can be stochastically stable, which means that the mixed strategy $z^{*}$ must be stochastically stable. From (6), we know that $z^{*}$ must also be convergence stable, which means $\mathrm{d} \delta(z) /\left.\mathrm{d} z\right|_{z=z^{*}}<0$.

The switch in the stability of the mixed strategy $z^{*}$ as $\kappa$ decreases indicates a change in the underlying game structure as $\kappa$ is varied. The mixed strategy in a coordination game without population structure $(\kappa=0)$ is an evolutionary repeller (i.e., not convergence stable) and separates the basins of attraction for the two boundary phenotypes, playing actions $r$ and $p$ with probability 1 , respectively. When the mixed strategy becomes stable as $\kappa$ decreases, the boundary strategies become unstable and the resulting game has the structure of a two-player hawk-dove (Maynard Smith and Price, 1973) or snowdrift game (Sugden, 1986). This can been in Fig. 5, which shows the location and stability of the mixed strategy $z^{*}$ and the stability of the boundary phenotypes, $z=0$ and $z=1$. For more than two players and $T=0.25$, we see that: (i) the mixed strategy is stable for values of $\kappa$ close to -1 and the boundaries $z=0$ and $z=1$ are repelling; (ii) the mixed strategy disappears for a window of $\kappa<0$ and $z=1$ becomes attracting; and (iii) the mixed strategy reappears as an unstable point for $\kappa \gtrsim-0.5$ and $z=0$ becomes attracting. This thus covers the generic twoplayer two-action game structures (e.g., Weibull, 1997); namely scenario (i) results in a hawk-dove type interaction, (ii) is a game with a dominant strategy, and (iii) is a coordination game.

As $T$ increases further ( $T=1.25$ in Fig. 5), this sequence of game structures changes. Values of $\kappa$ less than -0.5 result in the stability of $z=0$ and instability of $z=1$, which create a prisoner's dilemma interaction. As $\kappa$ increases above about -0.5 , the mixed strategy $z^{*}$ appears as an unstable point resulting in a coordination game. As $\kappa$ continues to increase, $z^{*}$ decreases, which means the basin of attraction of $r$ increases and at some point $r$ will become stochastically stable.

\section{Discussion}

Building from previous work on the evolution of continuous phenotypes in finite populations in evolutionary biology (Rousset, 2004; Champagnat and Lambert, 2007; Lehmann, 2012) and the effect of stochastic forces on the selection of strategies in games (Foster and Young, 1990; Blume, 1993; Ellison, 1993; Noldeke and Samuelson, 1993; Kandori et al., 1993; Young, 1993; Fudenberg et al., 2006), we have extended the concept of stochastic stability to continuously varying strategies. This enabled us to show how genetic relatedness (scaled to take local competition into account) generated by spatial structure and the size of interaction groups can effect the evolution of behavior in coordination games. Specifically, we find that the usual notion that selection results in the evolution of risk dominant strategies, regardless of whether they are payoff dominant, is only a special case for a specific value of relatedness. In general, positive relatedness (low local competition) increases the range of payoff values under which the payoff dominant strategy can evolve so that it can evolve even when it is not risk dominant. In contrast, negative relatedness (high local competition) results in more cases where the strategy that is not payoff dominant can evolve, even when it is not risk dominant. In large interaction groups ( $n$-player games where $n$ is large), this pattern holds except that negative relatedness can generate so much local competition that the outcome of the interaction resembles a hawk-dove or anti-coordination game in that a stable polymorphism between the two strategies emerges.

In game theory, stochastic stability has become a widely used approach because it reduces the number of potential solutions to a game (Nash equilibria) and provides a unique prediction for longterm evolution. Without such a mechanism to select among equilibria, which equilibrium is likely to be observed depends on initial conditions, which are often impossible to measure in real world scenarios (biological, economic, or otherwise). Using evolutionary metaphors to construct learning rules that determine how individuals change their actions over time, game theory models show that the stochastically stable action is the risk dominant one, both in panmictic populations (Kandori et al., 1993; Young, 1993) and in populations with spatial structure (Blume, 1993; Ellison, 1993). This stands in contrast to the results found here and in Fudenberg et al. (2006), where risk dominance is not equivalent to stochastic stability. A likely reason for this discrepancy is that in learning models, mistakes (the analog of mutation) cannot propagate in a population stochastically by faithful "reproduction". In stark contrast, a single mutation can lead to a population transitioning from one fitness peak to another owing to the interaction between selection and genetic drift. The probability of these transitions is taken into account in our model by the fact that the long-term distribution of phenotypes depends on the integral of the selection gradient (the "speed" of phenotypic change) over the whole range of possible phenotypes (see Eqs. (3) and (4)).

The key feature of coordination games, multiple ESSs and the problem of selection among the alternative adaptive peaks that they generate, has been underemphasized in the biological study of social behaviors. However, this phenomenon has long been appreciated in evolutionary theory, which has studied how populations evolve from one peak to another (i.e., "peak shifting" or "valley crossing"). This problem was emphasized in the shifting balance theory Wright $(1931,1932)$, which suggested the accumulation of complex adaptations can be catalyzed by population subdivision. This view is often contrasted with one often attributed to 

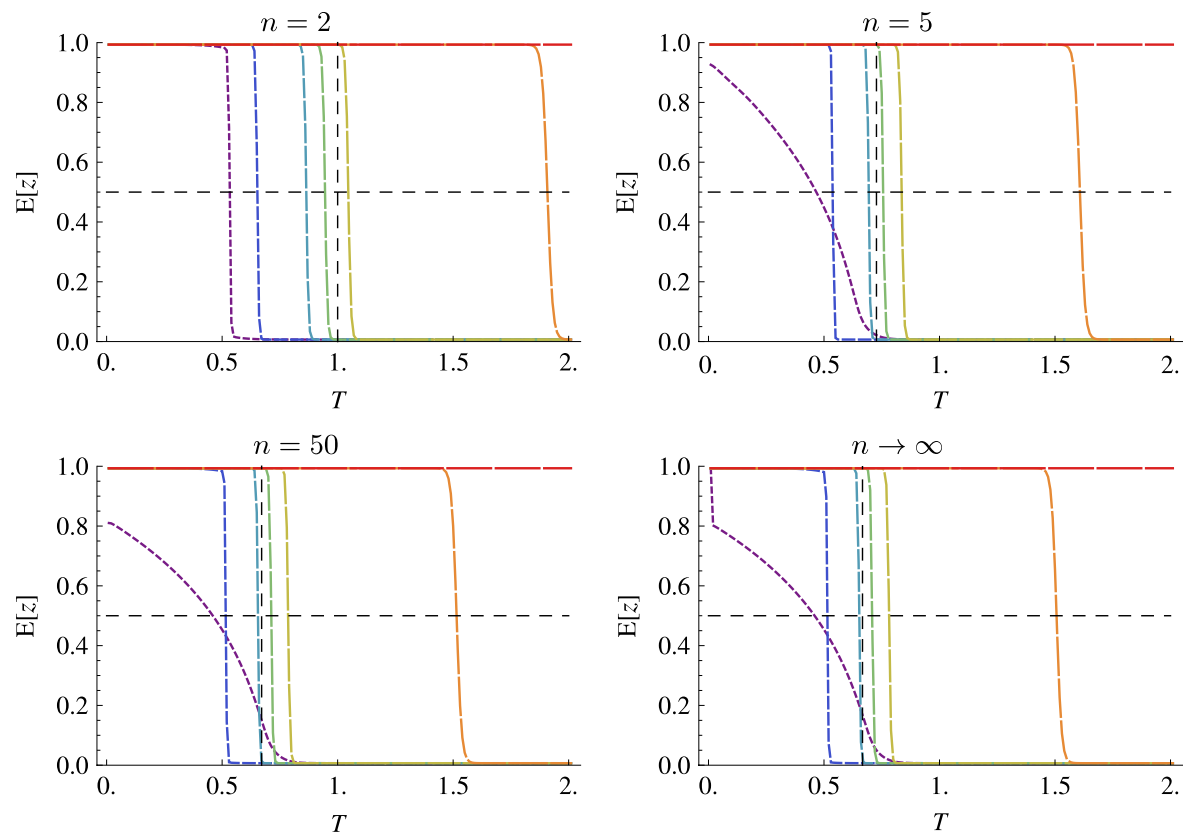

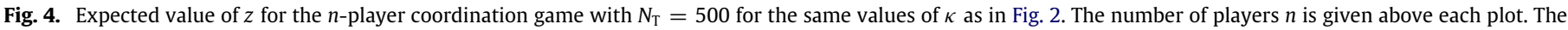

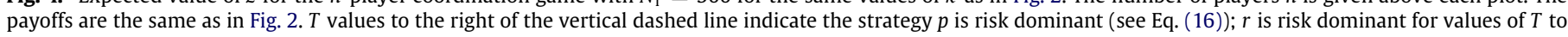
the left of the dashed line.
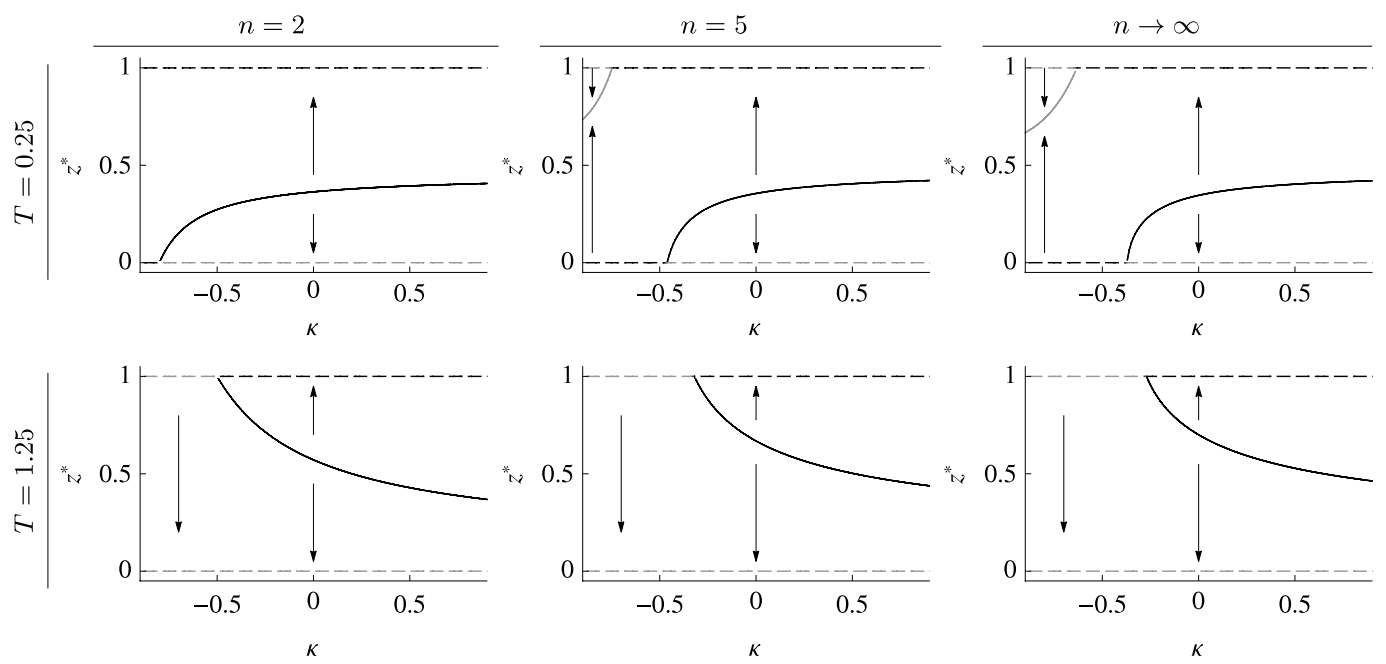

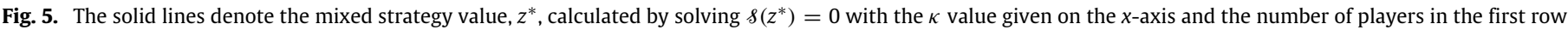

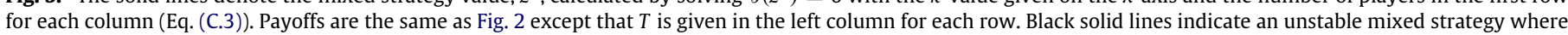

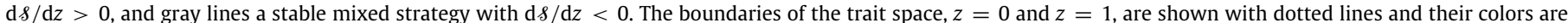

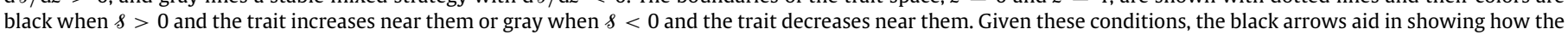
selection gradient would change the value of the play probability $z$ under a deterministic dynamic.

R. A. Fisher that adaptation occurs most easily in large well-mixed populations (Coyne et al., 1997; Wade and Goodnight, 1998; Frank, 2012). In light of these contrasting views, much of the work on this problem has focused on exploring the role of (effective) population size and genetic architecture (strength of epistasis or valley depth or "ruggedness", mutation rates, and recombination rate) on the rate of valley crossing (Barton and Rouhani, 1987; Iwasa et al., 2004; Weinreich and Chao, 2005; Handel and Rozen, 2009; Weissman et al., 2009; Lynch and Abegg, 2010; Weissman et al., 2010; Jain et al., 2011). Generically, this works suggests that the rate of valley crossing increases with population size as long as the valley is not too deep (Weissman et al., 2010). This pattern is consistent with our stochastic stability results that predict the long-term evolution of the highest fitness peak in the limit of very large total population size (expression (6)).
One key feature of our analysis is the extension of a twoplayer coordination game in a structured population to $n$-players. We find that varying the scaled relatedness $\kappa$ can lead to novel evolutionary outcomes (e.g., a stochastically stable polymorphism for highly negative relatedness) that are analogous to outcomes from other two-player games (e.g., the hawk-dove game). Our $n$-player interaction setting is a multiplayer matrix game, the study of which has been gaining interest in evolutionary biology (e.g., Motro, 1991; Bach et al., 2006; Hauert et al., 2006; Souza et al., 2009; Pacheco et al., 2009; Archetti, 2009; Gokhale and Traulsen, 2010; Peña, 2012; Kurokawa and Ihara, 2013). A general result from this work is that $n$-player matrix games have more complex dynamics than their two-player analogs. For example, many models study $n$-player social dilemmas where a threshold number of cooperators is needed for the production of group 
benefits (Bach et al., 2006; Archetti, 2009; Pacheco et al., 2009) or for sharing the costs of such production (Souza et al., 2009). Typically, these models find that no cooperation is stable for benefit to cost ratios $(b / c)$ below a certain threshold. Above this threshold, no cooperation is still stable, but two new polymorphic equilibria appear, one unstable and one stable. With too few initial cooperators, the population evolves towards no cooperation, whereas for a sufficient initial fraction, the frequency of cooperator reaches the stable polymorphism. Unlike these threshold games, the dynamics in our $n$-player game is directly analogous to one of the three generic two-player game types (games with a dominant strategy, a hawk-dove game with an interior stochastically stable state, and a stag hunt game with an interior unstable state) depending on the value of $\kappa$. This is ultimately due to the fact that there is (almost surely) a unique stochastically stable state. Thus, stochastic stability allows us to make a simple prediction for how spatial structure affects the evolutionary outcome independently of other nonlinearities in an $n$-player game.

Our analysis is another example in a significant body of work (see Frank, 1998 for standard examples) that illustrates how multiplayer games can be analyzed only in terms of pairwise (scaled) relatedness. This is ultimately due to the fact that small phenotypic deviations (small $\delta$ values) imply additive gene action (Rousset, 2004), which allows one to track the gradual change of mixed strategies by evaluating Hamilton's inclusive fitness effect (Hamilton, 1964). By contrast, in the analysis of pure strategies, fitness differences between the mutant and resident can involve nonlinear genetic effects, which requires the calculation of at least triplet relatedness coefficients in spatially structured populations (Lehmann et al., 2007c; Tarnita et al., 2009; Ohtsuki, 2010, 2012; Taylor and Maciejewski, 2012). Such payoff nonlinearities can produce complex dynamics, particularly in the case of $n$-player games. We find, however, that we can recover the same qualitative stochastic stability results for panmictic populations as that obtained from an analysis of pure strategies (see Section 3.1.3), and it would be interesting to investigate whether this result also holds for structured populations. More generally, it would be relevant to compare the qualitative outcomes of continuous and pure strategies across identical games as a concordance would allow a focus on continuous (mixed) strategies. An evolutionary analysis of continuous strategies is both simpler and maybe also more realistic since many, if not most, traits are quantitative (Lynch and Walsh, 1998).

As evolutionary models of behavior accumulate biological realism (e.g. large interaction groups and complex population structures), it becomes increasingly difficult to derive predictions simple enough to compare to real world data. Part of this difficulty arises from the possibility of multiple fitness peaks (multiple equilibria in games), which can exist even in simple social interactions. Although predicting how evolution proceeds in multipeaked landscapes is very difficult in general, the concept of stochastic stability provides a simple answer for the case of very large populations. In combination with standard results from evolutionary biology, stochastic stability can generate a simple and unique prediction for how behaviors evolve, even in populations with complex spatial structures and demographies where genetic relatedness may be important. We used these results to analyze the evolution of coordination games in groups of arbitrary size in this work, but all two-player or multiplayer game structures are amenable to such analysis. Thus, future work should be able to completely characterize the effect of population structure on the equilibria of $n$-player games and how this effect interacts with the size of the interaction group.

\section{Acknowledgments}

We thank Jorge Peña for useful comments on the paper. JV was supported by a SFI Omidyar Fellowship and by the National
Evolutionary Synthesis Center (NESCent) under NSF grant \#EF0423641. This work was supported by Swiss NSF grant PP00P3123344.

\section{Appendix A. Stochastically stable states}

Here, we show that any stochastically stable state $z$ belongs to $\mathcal{M}$, which is defined as the set of points maximizing the potential $\phi(x): \mathcal{M}=\left\{y: \phi(y)=\max _{z \in Z} \phi(z)\right\}$. In order to demonstrate this, we use the function

$\Delta \phi(z)=\phi(z)-\max _{z \in Z} \phi(z)$,

where $\Delta \phi(z)=0$ for $z \in \mathcal{M}$ and $\Delta \phi(z)<0$ for $z \notin \mathcal{M}$ (Sandholm, 2010). With this, we can write $\exp \left[2 N_{\mathrm{T}} \phi(z)\right]=$ $\exp \left[2 N_{\mathrm{T}} \Delta \phi(z)\right] \exp \left[2 N_{\mathrm{T}} \max _{z \in Z} \phi(z)\right]$ and substituting this into $p(z)$ from (3) gives

$\lim _{N_{\mathrm{T}} \rightarrow \infty} p(z)=\lim _{N_{\mathrm{T}} \rightarrow \infty} \frac{\exp \left[2 N_{\mathrm{T}} \Delta \phi(z)\right]}{\int_{l}^{r} \exp \left[2 N_{\mathrm{T}} \Delta \phi(y)\right] \mathrm{d} y}$,

where $l$ and $r$ are, respectively, the left and right boundaries of the phenotypic state space.

Suppose that $z \in \mathcal{M}$. Thus, $\lim _{N_{\mathrm{T}} \rightarrow \infty} p(z)=\lim _{N_{\mathrm{T}} \rightarrow \infty}\left(\int_{l}^{r} \exp \right.$ $\left.\left[2 N_{\mathrm{T}} \Delta \phi(y)\right] \mathrm{d} y\right)^{-1}$ since $\Delta \phi(z)=0$. If we assume that there are only a countable number of convergence stable states ( $\mathcal{M}$ is countable), the integral in the denominator converges to zero as $N_{\mathrm{T}} \rightarrow \infty$ and $\lim _{N_{\mathrm{T}} \rightarrow \infty} p(z)=\infty$.

Now, suppose that $z \notin \mathcal{M}$. For convenience, we rewrite $\lim _{N_{\mathrm{T}} \rightarrow \infty} p(z)$ as

$\lim _{N_{\mathrm{T}} \rightarrow \infty} p(z)=\frac{1}{\lim _{N_{\mathrm{T}} \rightarrow \infty} \int_{l}^{r} \exp \left[2 N_{\mathrm{T}}(\Delta \phi(y)-\Delta \phi(z))\right] \mathrm{d} y}$.

Given that $s(y)$ is continuous, which means that $\phi(z)$ and $\Delta \phi(z)$ are continuous, there exists an open interval around any $m \in \mathcal{M}$, called $\epsilon_{m}=\left\{\epsilon_{-}, \epsilon_{+}\right\}$with $l \leq \epsilon_{-}<\epsilon_{+} \leq r$, where $\Delta \phi(y)-$ $\Delta \phi(z)=\phi(y)-\phi(z)>0$ for all $y \in \epsilon_{m}$. Thus,

$\lim _{N_{\mathrm{T}} \rightarrow \infty}=\int_{\epsilon_{-}}^{\epsilon_{+}} \exp \left[2 N_{\mathrm{T}}(\Delta \phi(y)-\Delta \phi(z))\right] \mathrm{d} y=\infty$,

which means that the denominator of (A.3) is equal to infinity and $\lim _{N_{\mathrm{T}} \rightarrow \infty} p(z)=0$.

Putting together the $z \in \mathcal{M}$ and $z \notin \mathcal{M}$ cases, we can conclude that, as $N_{\mathrm{T}} \rightarrow \infty$, the distribution $p(z)$ consists of equal sized point masses at the points $z \in \mathcal{M}$ and zero elsewhere.

\section{Appendix B. Effect of population structure on $\kappa$ and $k$}

Here, we illustrate how different values of $\kappa$ and $k$ in Eq. (7) describe different levels of population structure and different underlying demographic assumptions in our model of local social interactions.

Let us first assume that evolution occurs in a panmictic population with semelparous individuals (synchronous reproduction where all individuals reproduce and die at the same time; that is, the Wright-Fisher reproductive scheme; Ewens, 2004). In this scenario, social behaviors affect only the fecundity of individuals and one has

$\kappa=-\frac{1}{N_{\mathrm{T}}-1}$

$k=\frac{N_{\mathrm{T}}-1}{N_{\mathrm{T}}}$.

(Rousset, 2004, Eq. (7.21)). The negative value of $\kappa$ stems from the fact that in a panmictic population, $\partial f\left(z_{\bullet}, z_{0}\right) / \partial z_{0}$ measures an increase in competition faced by the offspring of the focal individual with offspring of individuals affected by the behavior of the focal. The offspring of the focal compete with such offspring with probability $1 /\left(N_{\mathrm{T}}-1\right)$ so that $-1 /\left(N_{\mathrm{T}}-1\right)$ can be thought 
of as the relatedness among competing offspring in the population. The value $\kappa=-1 /\left(N_{\mathrm{T}}-1\right)$ actually holds more generally, whether reproduction occurs under the Wright-Fisher, Cannings, or Moran process and in the latter two cases whether social behaviors affect the fecundity or survival of adults (Lehmann and Rousset, 2012, Eqs. (9) and (11) by setting $t=0, \mathbf{k}=\mathbf{0}, H=0$, and $\delta_{\mathbf{0}, 0}=0$ because social effects are on patch neighbors only).

Suppose now the population consists of $n_{\mathrm{d}}$ groups each with $N$ individuals, and migration occurs at rate $m$ according to the island model of dispersal under a Wright-Fisher reproductive protocol. In this case, $\kappa$ and $k$ are also given by Eq. (B.1), but where $N_{\mathrm{T}}=n_{\mathrm{d}} N$ (Rousset, 2004, Eq. (7.21)). Hence, population structure does not affect the direction of evolution of the trait, a result first established analytically by Taylor (1992) in a population of infinite size $\left(N_{\mathrm{T}} \rightarrow\right.$ $\infty)$. When regulation occurs before dispersal in such a structured population, then Eq. (B.1) still applies but with $N_{\mathrm{T}}=N$ (Eq. (A.7) of Lehmann and Rousset, 2010, substituted into Eq. (6.23) of Rousset, 2004).

On the other hand, for a Moran reproductive protocol for an island model of dispersal with fecundity effects, one has

$$
\begin{aligned}
& \kappa=\frac{(1-m)-2 / n_{\mathrm{d}}}{[N+(1-m)]-2 / n_{\mathrm{d}}} \\
& k=\frac{[N+(1-m)]-2 / n_{\mathrm{d}}}{N^{2} n_{\mathrm{d}}}
\end{aligned}
$$

(Lehmann et al., 2007a, Eq. (32) pre-multiplied by $1 /\left(N n_{d}\right)$ ). Hence, for a Moran process, the migration rate affects the selection pressure, and the higher the migration rate, the lower the value of $\kappa$.

When the payoff function (Eq. (1)) affects the survival probability $s$ of individuals (instead of the fecundity), one has for the finite island model

$$
\begin{aligned}
& \kappa=-\frac{(1-m)(1-s)+2 s / n_{\mathrm{d}}-(1+s) / n_{\mathrm{d}}^{2}}{N\left(2-m(1-s)-2 / n_{\mathrm{d}}\right)-(1-m)(1-s)+(1+s) / n_{\mathrm{d}}^{2}-2 s / n_{\mathrm{d}}} \\
& k=s\left[1-\frac{(1-m)(1-s)+2 s / n_{\mathrm{d}}-(1+s) / n_{\mathrm{d}}^{2}}{N\left(2-m(1-s)-2 / n_{\mathrm{d}}\right)}\right],
\end{aligned}
$$

which is a situation where $\kappa$ tends to be always negative. Hence, individuals should decrease the survival of neighbors.

When whole groups form propagules by splitting at a rate $q$ that compete against each other and where individuals within groups migrate between groups at a rate $m$, one has

$$
\begin{aligned}
\kappa & =\frac{q-\left[2 q / n_{\mathrm{d}}+m /\left(N n_{\mathrm{d}}\right)\right]}{m\left(N n_{\mathrm{d}}-1\right) /\left(N n_{\mathrm{d}}\right)+q\left(N+n_{\mathrm{d}}-2\right) / n_{\mathrm{d}}} \\
k & =\frac{m\left(N n_{\mathrm{d}}-1\right)+N q\left(N+n_{\mathrm{d}}-2\right)}{2 N n_{\mathrm{d}}(m+N q)}
\end{aligned}
$$

(Lehmann et al., 2007b, Eqs. (A.20)-(A.42)), which can be made arbitrarily close to one when the migration rate tends to zero $(m \rightarrow$ 0 ) but groups compete against each other $(q>0)$. However, when $q \rightarrow 0$, one obtains $\kappa=-1 /\left(n_{\mathrm{d}} N-1\right)$ since competition occurs only at the level of the local group. Computing the expression $1 / \kappa$ gives Eq. (34) of Traulsen and Nowak (2006).

One can also take $z_{0}$ as the average phenotype of the nearest neighbors of a focal individual when individuals live on the nodes of a lattice $(N=1)$ and interact in a pairwise manner. In this case, under a Moran process of reproduction with stepping-stone dispersal at a rate $m$ to the $k$-nearest neighbors and fecundity effects of social behavior, one has

$$
\begin{aligned}
\kappa & =\frac{m-2 k / n_{\mathrm{d}}}{k(2-m)-2 k / n_{\mathrm{d}}} \\
k & =\frac{n_{\mathrm{d}}(2-m)-2}{n_{\mathrm{d}}^{2}},
\end{aligned}
$$

(Lehmann et al., 2007a, Eq. (32) pre-multiplied by $1 /\left(N n_{\mathrm{d}}\right)$ ). For $m=1$ and $k=2$, the expression $1 / \kappa$ then provides Eq. (4.4) of Ohtsuki and Nowak, 2006 for their "death-birth" process.

Finally, we consider interactions within groups in an arbitrarily structured homogeneous population with iteroparous reproduction and survival probability $s$ of an individual to the next generation. If one assumes strong population structure $(m \ll 1)$, then regardless of the dispersal kernel one has for fecundity effects

$\kappa=\frac{s[2-m(1+s)] / 2-(1+s) / n_{\mathrm{d}}}{N+s[2-m(1+s)] / 2-(1+s) / n_{\mathrm{d}}}$

$k=(1-s)\left\{1+s[2-m(1+s)] /(2 N)-(1+s) /\left(N n_{\mathrm{d}}\right)\right\}$,

(Lehmann and Rousset, 2012, Eq. (A.53), where for this model $s(z)=\tau_{\bullet} \partial f\left(z_{\bullet}, z_{0}\right) / \partial z_{\bullet}+\tau_{\mathbf{0}, 0} \partial f\left(z_{\bullet}, z_{0}\right) / \partial z_{0}$ in terms of the components of Eq. (A.53) and where the "1" in $\tau_{\mathbf{0}, 0}$ needs to be suppressed to account for the fact that $\partial f\left(z_{\bullet}, z_{0}\right) / \partial z_{0}$ measures the effects of others). In the case of survival effects, we have for strong population structure

$\kappa=-\frac{(1-s)[2-m(1+s)] / 2+(1+s) / n_{\mathrm{d}}}{2 N-(1-s)[2-m(1+s)] / 2-(1+s) / n_{\mathrm{d}}}$

$k=s\left\{1-(1-s)[2-m(1+s)] /(4 N)-(1+s) /\left(2 N n_{\mathrm{d}}\right)\right\}$

(Lehmann and Rousset, 2012, Eq. (A.55) where for this model $s(z)=\tau_{\bullet} \partial f\left(z_{\bullet}, z_{0}\right) / \partial z_{\bullet}+\tau_{\mathbf{0}, 0} \partial f\left(z_{\bullet}, z_{0}\right) / \partial z_{0}$ in terms of the components of Eq. (A.53) and where the "1" in $\tau_{\mathbf{0}, 0}$ needs to be suppressed to account for $\partial f\left(z_{\bullet}, z_{0}\right) / \partial z_{0}$ measuring the effects of others).

\section{Appendix C. $n$-player interactions}

In order to derive intuition for the $n$-player case, we extend the two-player coordination game to $n$ players in the following simple way, which is allowed by our trait substitution model assumptions (namely, weak selection and mutation, with mutation much weaker than effective selection strength; see Champagnat, 2006). Suppose that when the focal individual chooses action $r$, its payoff is altered by a factor $\gamma$ for every one of the other $n-1$ individuals who chooses action $p$. When the focal chooses action $p$, its payoff is altered by a factor $\lambda$ for every other individual who chooses action $r$. We assume that the other individuals in the population each independently choose action $r$ with the same probability $z_{0}$ (i.e., the other individuals have average phenotype $z_{0}$, which matches the assumption of previous work, Motro, 1991; Bach et al., 2006). If $\gamma$ and $\lambda$ alter payoffs multiplicatively, the expected payoff of a mutant individual who chooses action $r$ is

$$
\begin{aligned}
1 & +R \sum_{x=0}^{n-1}\left(\begin{array}{c}
n-1 \\
x
\end{array}\right) \gamma^{x}\left(1-z_{0}\right)^{x} z_{0}^{n-1-x} \\
& =1+R\left(z_{0}+\gamma\left(1-z_{0}\right)\right)^{n-1}=1+R M_{r}\left(z_{0}\right)^{n-1},
\end{aligned}
$$

and the expected payoff of the focal individual when it chooses $p$ is

$$
\begin{aligned}
1 & +P \sum_{x=0}^{n-1}\left(\begin{array}{c}
n-1 \\
x
\end{array}\right) \lambda^{x} z_{0}^{x}\left(1-z_{0}\right)^{n-1-x} \\
& =1+P\left(\lambda z_{0}+1-z_{0}\right)^{n-1}=1+P M_{p}\left(z_{0}\right)^{n-1} .
\end{aligned}
$$

If the focal individual chooses action $r$ with probability $z_{\bullet}$, then, using Eqs. (C.1) and (C.2), its payoff is given by Eq. (14) in the main text. We set $\gamma=(S / R)^{1 /(n-1)}$ and $\lambda=(T / P)^{1 /(n-1)}$ so that the pure-strategy outcomes match the two player case; i.e., the focal obtains payoff $1+R$ when it and all its partners always play $r, 1+S$ when it always plays $r$ and its partners always play $p, 1+T$ when it always plays $p$ and its partners always play $r$, and $1+P$ when 
it and its partners always play $p$. This also results in Eqs. (C.1) and (C.2) simplifying correctly for the two-player case $(n=2)$ for pure and mixed strategies.

Given the payoff in (14), we can derive the fixation probability perturbation $f(z)$ by substituting Eqs. (C.1) and (C.2) into the selection gradient in (7):

$$
\begin{aligned}
s(z)= & \frac{k}{f(z, z)}\left(R M_{r}(z)^{n-1}-P M_{p}(z)^{n-1}+\kappa(n-1)\right. \\
& \times\left[R z\left(1-\left(\frac{S}{R}\right)^{\frac{1}{n-1}}\right) M_{r}(z)^{n-2}-P(1-z)\right. \\
& \left.\left.\times\left(1-\left(\frac{T}{P}\right)^{\frac{1}{n-1}}\right) M_{p}(z)^{n-2}\right]\right) .
\end{aligned}
$$

We can then integrate $s(z)$ numerically using Eqs. (3) and (4) to obtain the expected value of $z$ at stationarity, which is plotted in Fig. 4.

In order to calculate the mixed strategy Nash equilibrium, $z^{*}$, of the $n$-player game, we determine when the expected payoffs of using actions $r$ and $p$ are equal, which is equivalent to determining when the payoff of the focal individual is independent of $z_{\bullet}$ (Motro, 1991; Bach et al., 2006). For $\kappa=0$, this occurs when

$$
R M_{r}\left(z^{*}\right)^{n-1}=P M_{p}\left(z^{*}\right)^{n-1} .
$$

Solving for $z^{*}$, we obtain Eq. (15) in the main text. As is described in the main text, we can then use the location of the mixed strategy to determine which equilibrium, $z=1$ or $z=0$, is risk dominant.

In generating Eqs. (C.1) and (C.2), we could alternatively assume that $\gamma$ and $\lambda$ alter payoff in an additive way so that $\gamma^{x}$ is replaced by $1+\gamma x$ in (C.1) and $\lambda^{x}$ is replaced by $1+\lambda x$ in (C.2). We again choose $\gamma$ and $\lambda$ such that pure strategy payoffs match the twoplayer case regardless of $n$. This yields $\gamma=(S-R) /(R(n-1))$ and $\lambda=(T-P) /(P(n-1))$. When these values of $\gamma$ and $\lambda$ are used to generate the payoff of a focal individual, the equivalent of Eq. (14), the resulting expression simplifies to the two-player case in Eq. (1). Thus, additive $n$-player interactions generate the same long-term results as two-player interactions.

\section{References}

Akcay, E., Van Cleve, J., 2012. Behavioral responses in structured populations pave the way to group optimality. Amer. Nat. 179, 257-269.

Alger, I., Weibull, J.W., 2012. A generalization of Hamilton's rule-love others how much? J. Theoret. Biol. 299, 42-54. Evolution of Cooperation.

AllenB., Nowak, DieckmannU., 2013. Adaptive dynamics with interaction structure. Amer. Nat. 181, E139-E163. ArticleType: research-article/Full publication date: June 2013/Copyright @2013 The University of Chicago.

Archetti, M., 2009. The volunteer's dilemma and the optimal size of a social group. J. Theoret. Biol. 261, 475-480.

Bach, L., Helvik, T., Christiansen, F., 2006. The evolution of $n$-player cooperationthreshold games and ESS bifurcations. J. Theoret. Biol. 238, 426-434.

Barton, N.H., Rouhani, S., 1987. The frequency of shifts between alternative equilibria. J. Theoret. Biol. 125, 397-418.

Binmore, K.G., 2007. Playing for Real: A Text on Game Theory. Oxford University Press, Oxford.

Binmore, K.G., Samuelson, L., Vaughan, R., 1995. Musical chairs: modeling noisy evolution. Games Econom. Behav. 11, 1-35.

Blume, L.E., 1993. The statistical mechanics of strategic interaction. Games Econom. Behav. 5, 387-424.

Boesch, C., 1994. Cooperative hunting in wild chimpanzees. Anim. Behav. 48, 653-667.

Boesch, C., 2002. Cooperative hunting roles among Taï chimpanzees. Hum. Nat. 13, 27-46.

Bürger, R., 2000. The Mathematical Theory of Selection, Recombination, and Mutation. John Wiley and Sons, New York.

Champagnat, N., 2006. A microscopic interpretation for adaptive dynamics trait substitution sequence models. Stochastic Process. Appl. 116, 1127-1160.

Champagnat, N., Ferrière, R., Méléard, S., 2006. Unifying evolutionary dynamics: from individual stochastic processes to macroscopic models. Theor. Popul. Biol. 69, 297-321.
Champagnat, N., Lambert, A., 2007. Evolution of discrete populations and the canonical diffusion of adaptive dynamics. Ann. Appl. Probab. 17, 102-155.

Christiansen, F.B., 1991. On conditions for evolutionary stability for a continuously varying character. Amer. Nat. 138, 37-50.

Coyne, J.A., Barton, N.H., Turelli, M., 1997. Perspective: a critique of Sewall Wright's shifting balance theory of evolution. Evolution 51, 643-671.

Crawford, V.P., 1991. An "evolutionary" interpretation of Van Huyck, Battalio, and Beil's experimental results on coordination. Games Econom. Behav. 3, 25-59.

Ellison, G., 1993. Learning, local interaction, and coordination. Econometrica 61 1047-1071.

Eshel, I., 1983. Evolutionary and continuous stability. J. Theoret. Biol. 103, 99-111.

Eshel, I., 1996. On the changing concept of evolutionary population stability as a reflection of a changing point of view in the quantitative theory of evolution. J. Math. Biol. 34, 485-510.

Eshel, I., Feldman, M.W., Bergman, A., 1998. Long-term evolution, short-term evolution, and population genetic theory. J. Theoret. Biol. 191, 391-396.

Ewens, W.J., 2004. Mathematical Population Genetics. Springer-Verlag, New York.

Foster, D., Young, H.P., 1990. Stochastic evolutionary game dynamics. Theor. Popul. Biol. 38, 219-232.

Frank, S.A., 1998. Foundations of Social Evolution. Princeton University Press, Princeton, NJ.

Frank, S.A., 2012. Wright's adaptive landscape versus Fisher's fundamental theorem. In: Svensson, E., Calsbeek, R. (Eds.), The Adapative Landscape in Evolutionary Biology. Oxford University Press, New York, NY, pp. 41-57.

Fudenberg, D., Harris, C., 1992. Evolutionary dynamics with aggregate shocks. J. Econom. Theory 57, 420-441.

Fudenberg, D., Nowak, M.A., Taylor, C., Imhof, L.A., 2006. Evolutionary game dynamics in finite populations with strong selection and weak mutation. Theor. Popul. Biol. 70, 352-363.

Gardiner, C.W., 2009. Stochastic Methods, fourth ed. Springer-Verlag, Berlin.

Gardner, A., 2010. Sex-biased dispersal of adults mediates the evolution of altruism among juveniles. J. Theoret. Biol. 262, 339-345.

Geritz, S.A.H., Kisdi, E., Meszéna, G., Metz, J.A.J., 1998. Evolutionarily singular strategies and the adaptive growth and branching of the evolutionary tree. Evol. Ecol. 12, 35-57.

Gillespie, J.H., 1991. The Causes of Molecular Evolution. Oxford University Press, Oxford.

Gokhale, C.S., Traulsen, A., 2010. Evolutionary games in the multiverse. Proc. Natl. Acad. Sci. USA 107, 5500-5504.

Grafen, A., Archetti, M., 2008. Natural selection of altruism in inelastic viscous homogeneous populations. J. Theoret. Biol. 252, 694-710.

Hamilton, W.D., 1964. The genetical evolution of social behaviour. I. J. Theoret. Biol. 7, 1-16.

Hammerstein, P., 1996. Darwinian adaptation, population genetics and the streetcar theory of evolution. J. Math. Biol. 34, 511-532.

Handel, A., Rozen, D., 2009. The impact of population size on the evolution of asexual microbes on smooth versus rugged fitness landscapes. BMC Evol. Biol. $9,236$.

Harsanyi, J.C., Selten, R., 1988. A General Theory of Equilibrium Selection in Games. MIT Press, Cambridge, Mass.

Hauert, C., Michor, F., Nowak, M.A., Doebeli, M., 2006. Synergy and discounting of cooperation in social dilemmas. J. Theoret. Biol. 239, 195-202.

Hofbauer, J., Sigmund, K., 2003. Evolutionary game dynamics. Bull. Amer. Math. Soc. 40, 479-519 (electronic).

Iwasa, Y., 1988. Free fitness that always increases in evolution. J. Theoret. Biol. 135, $265-281$.

Iwasa, Y., Michor, F., Nowak, M.A., 2004. Stochastic tunnels in evolutionary dynamics. Genetics 166, 1571-1579.

Jain, K., Krug, J., Park, S.-C., 2011. Evolutionary advantage of small populations on complex fitness landscapes. Evolution 65, 1945-1955.

Kandori, M., Mailath, G., Rob, R., 1993. Learning, mutation, and long-run equilibria in games. Econometrica 61, 29-56.

Karlin, S., Taylor, H.M., 1981. A Second Course in Stochastic Processes. Academic Press, San Diego.

Kimura, M., 1965. A stochastic model concerning the maintenance of genetic variability in quantitative character. Proc. Natl. Acad. Sci. USA 54, 731-736.

Kimura, M., 1971. Theoretical foundation of population genetics at the molecular level. Theor. Popul. Biol. 2, 174-208.

Kurokawa, S., Ihara, Y., 2013. Evolution of social behavior in finite populations: a payoff transformation in general-player games and its implications. Theor. Popul. Biol. 84, 1-8.

Lehmann, L., 2012. The stationary distribution of a continuously varying strategy in a class-structured population under mutation-selection-drift balance. J. Evol. Biol. 25, 770-787.

Lehmann, L., Keller, L., Sumpter, D., 2007a. The evolution of helping and harming on graphs: the return of the inclusive fitness effect. J. Evol. Biol. 20, 2284-2229.

Lehmann, L., Keller, L., West, S.A., Roze, D., 2007b. Group selection and kin selection: two concepts but one process. Proc. Natl. Acad. Sci. USA 104, 6736-6739.

Lehmann, L., Rousset, F., 2010. How life history and demography promote or inhibit the evolution of helping behaviours. Phil. Trans. R. Soc. Ser. B 365, 2599-2617.

Lehmann, L., Rousset, F., 2012. The evolution of social discounting in hierarchically clustered populations. Mol. Ecol. 21, 447-471.

Lehmann, L., Rousset, F., Roze, D., Keller, L., 2007c. Strong reciprocity or strong ferocity? A population genetic view of the evolution of altruistic punishment. Amer. Nat. 170, 21-36.

Leimar, O., 2009. Multidimensional convergence stability. Evol. Ecol. Res. 11, 191-208 
Lessard, S., 1990. Evolutionary stability: one concept, several meanings. Theor Popul. Biol. 37, 159-170.

Lynch, M., Abegg, A., 2010. The rate of establishment of complex adaptations. Mol. Biol. Evol. 27, 1404-1414.

Lynch, M., Walsh, B., 1998. Genetics and Analysis of Quantitative Traits. Sinauer, Massachusetts.

Malécot, G., 1975. Heterozygosity and relationship in regularly subdivided populations. Theor. Popul. Biol. 8, 212-241.

Maynard Smith, J., Price, G.R., 1973. The logic of animal conflict. Nature 246, 15-18

Metz, J.J., Geritz, S.A.H., Meszéna, G., Jacobs, F.J.A., van Heerwaarden, J., 1996 Adaptive dynamics: a geometrical study of the consequences of nearly faithful reproduction. In: van Strien, S.J., Verduyn Lunel, S.M. (Eds.), Stochastic and Spatial Structures of Dynamical Systems. North-Holland, Amsterdam, pp. 183-231

Moran, P.A.P., 1958. Random processes in genetics. Math. Proc. Cambridge Philos. Soc. $54,60-71$.

Motro, U., 1991. Co-operation and defection: Playing the field and the ESS J. Theoret. Biol. 151, 145-154

Noldeke, G., Samuelson, L., 1993. An evolutionary analysis of backward and forward induction. Games Econom. Behav. 5, 425-454

Ohtsuki, H., 2010. Evolutionary games in Wright's island model: kin selection meets evolutionary game theory. Evolution 64, 3344-3353.

Ohtsuki, H., 2012. Does synergy rescue the evolution of cooperation? An analysis for homogeneous populations with non-overlapping generations. J. Theoret. Biol. 307, 20-28.

Ohtsuki, H., Nowak, M.A., 2006. Evolutionary games on cycles. Proc. R. Soc. Ser. B 273, 2249-2256.

Pacheco, J.M., Santos, F.C., Souza, M.O., Skyrms, B., 2009. Evolutionary dynamics of collective action in N-person stag hunt dilemmas. Proc. R. Soc. Ser. B 276 $315-321$

Peña, J., 2012. Group-size diversity in public goods games. Evolution 66, 623-636.

Queller, D.C., 1994. Genetic relatedness in viscous populations. Evol. Ecol. 8, 70-73.

Rodrigues, A.M.M., Gardner, A., 2012. Evolution of helping and harming in heterogeneous populations. Evolution 66, 2065-2079.

Rousset, F., 2003. A minimal derivation of convergence stability measures. J. Theoret. Biol. 221, 665-668.

Rousset, F., 2004. Genetic Structure and Selection in Subdivided Populations Princeton University Press, Princeton, NJ.

Rousset, F., 2006. Separation of time scales, fixation probabilities and convergence to evolutionarily stable states under isolation by distance. Theor. Popul. Biol. 69, 165-179.

Rousset, F., Ronce, O., 2004. Inclusive fitness for traits affecting metapopulation demography. Theor. Popul. Biol. 65, 127-141.
Sandholm, W.H., 2010. Orders of limits for stationary distributions, stochastic dominance, and stochastic stability. Theor. Econ. 5, 1-26.

Sawyer, S., Felsenstein, J., 1983. Isolation by distance in a hierarchically clustered population. J. Appl. Probab. 20, 1-10.

Schaffer, M.E., 1988. Evolutionarily stable strategies for a finite population and a variable contest size. J. Theoret. Biol. 132, 469-478.

Sella, G., 2009. An exact steady state solution of Fisher's geometric model and other models. Theor. Popul. Biol. 75, 30-34.

Sella, G., Hirsh, A.E., 2005. The application of statistical physics to evolutionary biology. Proc. Natl. Acad. Sci. USA 102, 9541-9546.

Skyrms, B., 2001. The stag hunt. Proc. Adresses Am. Philos. Assoc. 75, 31-41.

Souza, M.O., Pacheco, J.M., Santos, F.C., 2009. Evolution of cooperation under N-person snowdrift games. J. Theoret. Biol. 260, 581-588.

Sugden, R., 1986. The Economics of Rights, Co-Operation, and Welfare. Basil Blackwell, Oxford.

Tarnita, C.E., Ohtsuki, H., Antal, T., Fu, F., Nowak, M.A., 2009. Strategy selection in structured populations. J. Theoret. Biol. 259, 570-581.

Taylor, P.D., 1992. Altruism in viscous populations-an inclusive fitness model. Evol. Ecol. 6, 352-356.

Taylor, P.D., Jonker, L.B., 1978. Evolutionary stable strategies and game dynamics. Math. Biosci. 40, 145-156.

Taylor, P.D., Maciejewski, W., 2012. An inclusive fitness analysis of synergistic interactions in structured populations. Proc. R. Soc. Ser. B 279, 4596-4603.

Traulsen, A., Nowak, M., 2006. Evolution of cooperation by multilevel selection. Proc. Natl. Acad. Sci. USA 103, 10952-10955.

Van Dyken, J.D., 2010. The components of kin competition. Evolution.

Wade, M.J., Goodnight, C.J., 1998. Perspective: The theories of Fisher and Wright in the context of metapopulations: When nature does many small experiments. Evolution 52, 1537-1553.

Weibull, J.W., 1997. Evolutionary Game Theory. MIT Press, Cambridge, MA

Weinreich, D.M., Chao, L., 2005. Rapid evolutionary escape by large populations from local fitness peaks is likely in nature. Evolution 59, 1175-1182.

Weissman, D.B., Desai, M.M., Fisher, D.S., Feldman, M.W., 2009. The rate at which asexual populations cross fitness valleys. Theor. Popul. Biol. 75, 286-300.

Weissman, D.B., Feldman, M.W., Fisher, D.S., 2010. The rate of fitness-valley crossing in sexual populations. Genetics $186,1389-1410$

Wild, G., Taylor, P., 2004. Fitness and evolutionary stability in game theoric models of finite populations. Proc. R. Soc. Ser. B 271, 2345-2349.

Wright, S., 1931. Evolution in Mendelian populations. Genetics 16, 97-159.

Wright, S., 1932. The roles of mutation, inbreeding, crossbreeding and selection in evolution. In: Proceedings of the Sixth International Congress on Genetics, Vol. 1, pp. 356-366

Young, H.P., 1993. The evolution of conventions. Econometrica 61, 57-84. 